\title{
POWER SAVING MECHANISM FOR HYBRID ROUTING PROTOCOL USING SCHEDULING TECHNIQUE
}

\author{
Mohanavel $^{1}$, G. Ravi G ${ }^{2}$ \\ ${ }^{1} P G$ Student, M.E-communication Systems, Sona College of Technology, Tamil Nadu, India \\ ${ }^{2}$ Assistant Professor, ECE-PG, Sona college of Technology, TamilNadu, India
}

\begin{abstract}
Mobile ad hoc networks are infrastructure less network which are composed of wireless nodes of limited battery constraints with mobility and communication capabilities. Energy management is a challenging approach in such kind of wireless networks. It deals with controlling the battery discharge, adjusting the transmission power of the nodes, and scheduling of power source so as to reduce the energy consumption of individual nodes as much as possible. This paper proposes an Energy management algorithm for Zone Routing Protocol using the extended energy conservation technique called AFECA (Adaptive Fidelity Energy Conservation Algorithm) and Energy consumption is further optimized by using Distance based energy conservation algorithm by taking variable transmission range into consideration. These Energy saving Algorithms are incorporated with well-known hybrid routing protocol called Zone Routing Protocol to optimize the utilization of energy. The performance parameters such as Energy, throughput, delay, of modified ZRP are compared with ZRP using NS-2, which shows that modified ZRP is well suited and an efficient protocol.
\end{abstract}

Keywords: AFECA, Distance Based Energy Conservation, Energy management, Mobile Ad hoc network, Transmission Power, ZRP.

\section{INTRODUCTION}

MANET (Mobile Ad hoc Network) composed of group of nodes with limited battery constraints where a node itself functions as a router instead of requiring an additional network component for routing process. It attempts to provide communication during some critical applications like emergency rescue operations, military applications etc, where no network coverage is available. The communication link between nodes relies on neighbor nodes and these nodes are responsible for routing of information from source to destination. If these information or data packet has to be delivered successfully, the nodes in this network must be consistent for long period of time. In order to make the nodes as consistent as long as possible, the source for these nodes must be properly utilized or it has to be properly managed. Battery utilization in ad hoc networks determines the life time of the network hence the battery power must be properly discharged. A suitable routing protocol which helps to establish routes and accomplish packet delivery process among randomly distributed nodes. Based on the behavior of routing, the routing protocol is primarily classified in to three types as given below.

1. Pro Active routing protocol (Table Driven Approach): Each node will proactively maintains a table which keeps information needed for routing process by periodically transmitting Beacon messages to it neighbor nodes. Examples of such protocols are Destination Sequenced Distance Vector routing (DSDV), Optimized Link State Routing (OLSR) etc.
2. Reactive Routing protocol (On demand Routing Protocol): Nodes does not maintain updated routing table at every instant of time. It establish network link whenever the connection is needed. Examples of such protocols are AODV, DSR, etc.

3. Hybrid Routing Protocol: This protocol comprises of both the Pro Active and Reactive Mechanisms. Examples for such protocols are ZRP, CEDAR, etc.

\section{RELATED WORKS.}

Many researchers have been carried out in recent years which focus to develop several energy conservation algorithms [1] - [9]. This led to develop Enhanced Transmission Power Control Mechanism (ETPCM) [2], is an approach which calculates the required transmission power of the node based on RSSI (Received Signal Strength Indication). The calculation of transmission power for all nodes using RSSI will increase delay. In this case it is difficult to find the signal strength of non-neighbor nodes.

Minimum Battery Cost Routing [3] which estimates the residual energy of individual nodes and retain this to select suitable path for routing process. In some cases this routing may lead selection of routes with less power nodes which result in path damage.

Modified Zone Routing Protocol based on Alternate Zone creation process [4] has utilized alternate Zones of various power levels to reduce the wastage of transmission power in case of absence of peripheral nodes in Reactive mechanism. 
It creates alternate zones when peripheral nodes are not found hence it require re-establishment of routes and routing update would be necessary so it takes more time to establish routes and accomplish packet delivery process when alternate Zones are created.

Energy Efficient MANET routing [6] proposed a novel approach based on AFECA and Span which eliminates multiple retransmission of request by coordinator node selection and overhearing process. The nodes overhear data packets too it leads some nodes to act as a malicious node.

\section{MODIFIED ZONE ROUTING PROTOCOL}

Zone Routing Protocol is an example for Hybrid routing protocol which consists of both Proactive (IARP) and reactive mechanism (IERP). Each node in this network covers a Zone of Radius $\mathrm{R}$ as shown in fig 1 . It invokes proactive mechanism (IARP) when the destination is covered by the Zone region of source node and invokes Reactive mechanism (IERP) when the destination is not covered by the Zone region of source node. Each node in this network maintains routing table by periodically broadcasting beacon messages to its neighbors with the help of Neighbor Discovery Protocol (NDP). Since it is a Distributed protocol, broadcasting of multiple queries leads to redundancy. Such redundancy can be eliminated by using suitable query control mechanism. It drops the query to the particular node which has been already covered by that query with same sequence.

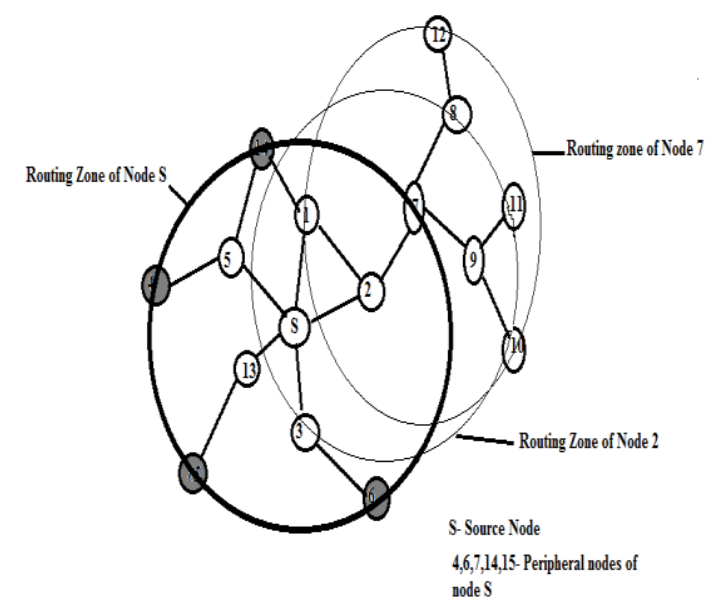

Fig 1: Concept of Zone Routing Protocol (ZRP)

Consider the network in fig-1, node $\mathrm{S}$ is the source node and the node $S$ has the data packet to send to node 12 . The radius of the zone is $\mathrm{R}=2$ hops. By using the routing table provided by IARP the source node checks the destination whether it is present within its zone region. Since it does not find the destination in its routing table it forwards the query using IERP to the peripheral nodes depicted as shaded circle in fig 1. Each peripheral node checks the destination in its routing table. Now the nodes 4, 6, 14 and 15 do not find the destination in its routing table so it repeats the border casting process. The node 7 found the destination in its routing table so it sends back reply to the source node $(\mathrm{S})$.
In this paper we have proposed an energy conservation algorithm for ZRP which focuses to reduce utilization of unwanted energy by proper scheduling and effective utilization of transmission power. The scheduling process involves assigning of three periodic time slots such as $T_{s}, T_{l}$, $\mathrm{T}_{\mathrm{a}}$, to all nodes in order to conserve the energy of individual nodes as much as possible. In accordance with AFECA technique every node in ZRP are operated with effective sleep schedule approach as shown in fig 2 . The effective sleep schedule approach aims to put the node in sleep mode for a pre assigned time period $\left(\mathrm{T}_{\mathrm{s}}\right)$ when it is not in use. As soon as a node finds that no traffic or request from its neighbor during its listening period $\left(T_{1}\right)$ it switches over to sleep mode. In case if it finds any messages or traffic from its neighbors it immediately processes the request by entering in to active mode $\left(\mathrm{T}_{\mathrm{a}}\right)$.

$\mathrm{T}_{1}=\mathrm{It}$ is the time over which the node Listen for traffic.

$\mathrm{T}_{\mathrm{s}}=\mathrm{It}$ is the time over which the node is in Sleep mode.

$\mathrm{T}_{\mathrm{a}}=\mathrm{It}$ is the time over which the node participates in Active Packet Delivery Process.

In order to ensure the successful delivery of data packets, repeated transmission of requests to $\mathrm{K}+1$ times would be necessary. This ensures that a node will reply for any one of the $\mathrm{K}+1$ request when it is in sleep mode. This may slightly increase overhead in the network. Once the node wakes up it will not give much impact on latency and overhead.

The time interval for sleep mode has been increased by the factor which is proportional to the density of neighbor nodes.

$$
\mathrm{T}_{\mathrm{SA}}=\operatorname{Random}(1, \mathrm{~N}) * \mathrm{~T}_{\mathrm{s}}
$$

Where $\mathrm{Ts}=\mathrm{K}^{*} \mathrm{~T}_{1}$, $\mathrm{N}=$ Number of nodes, $\mathrm{T}_{\mathrm{SA}}=$ new sleep time

Since it is an extended energy conservation technique we need to take node density into consideration. A node calculates its node density by tracking the number of packets seen during the listening time.

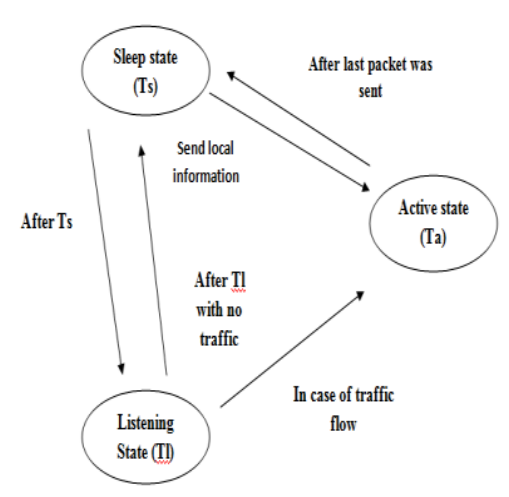

Fig 2: State Diagram for sleep Schedule Approach 
Active time of a node depends upon the length of the data packet that has to be delivered. A node in this network will be active until it finds no more packets to be processed. Once it detects that no more packet waiting in the queue, it allows the node to sleep for a particular period of time (Ts). The node wakes up from sleep mode after the time period (Ts) gets over.

Consider a network consist of 15 nodes of radius 2 hops as shown in Fig 3. This shows how the nodes are responsible for sleep schedule approach. In this node $S$ is Source and node 12 is destination. When the source $\mathrm{S}$ has the data to deliver to the destination, node $\mathrm{S}$ checks the destination in its routing table and it found that the destination node is not covered by the source Zone region so it follows IERP. Node $S$ broadcast request to its peripheral nodes (nodes 4, 6, 7, 14, 15) directly by using BRP (Border cast Resolution Protocol). As soon as the peripheral node 7 finds the destination in its routing table, it send reply back to the source node $\mathrm{S}$.

The nodes $\mathrm{S}, 2,7,8$, and 12 are involved in this process and the remaining nodes are not participated in this process so it goes to sleep mode or low power mode indicated as a grey circle in fig 3 . These nodes conserve their energy by switching into sleep mode when no traffic occurs.

Along with the scheduling approach a suitable technique has been incorporated to further optimize the power by taking distance between source and destination in to consideration. This algorithm helps to decide the sufficient energy level for delivering of data packet to the particular destination based on the hop distance. It is based on "Distance based energy conservation algorithm". A node adjusts its transmission power based on the distance

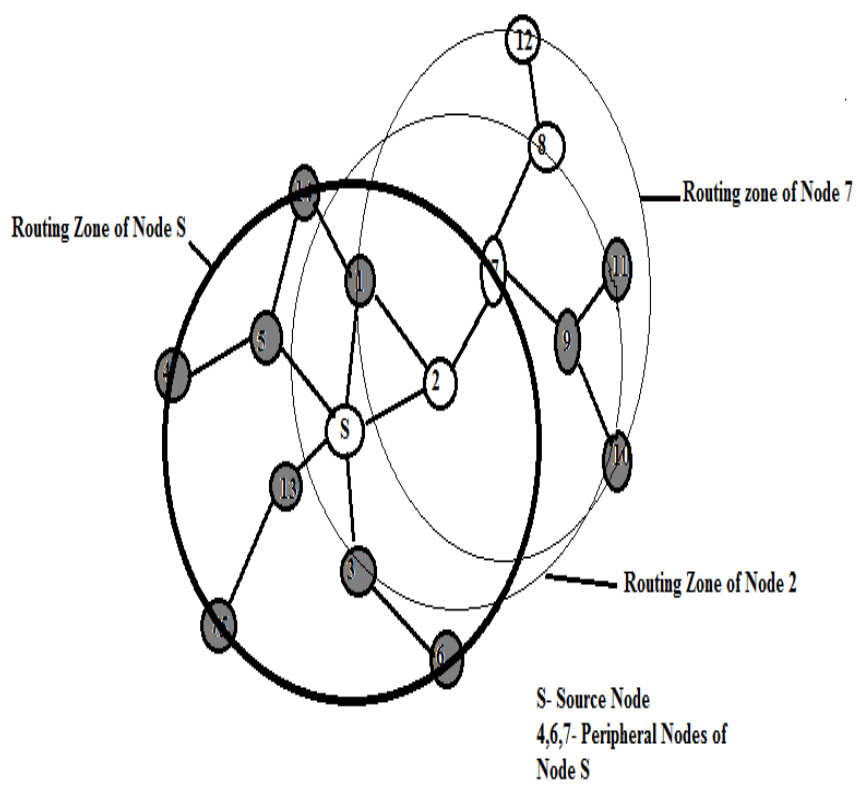

Fig 3: Process diagram for sleep schedule approach. between the corresponding node and the node it wish to deliver the data packet. Hence the destination node which is nearer to the source node would require less energy than the node which is far away from the source node. The distance and corresponding power levels can be calculated using Frris relation.

The various distances and corresponding power levels are given in table 1 .

Table 1

\begin{tabular}{|l|l|}
\hline $\begin{array}{l}\text { Distance Between Source } \\
\text { and Destination (M) }\end{array}$ & $\begin{array}{l}\text { Transmission power } \\
\text { level in joules }\end{array}$ \\
\hline Distance $<50$ & 0.09 \\
$50<$ Distance $<80$ & 0.12 \\
$80<$ Distance $<100$ & 0.15 \\
Distance $>100$ & 0.2 \\
\hline
\end{tabular}

Consider the diagram shown in fig 4, this shows how transmission power of the source node is adjusted based on the distance between source and the corresponding nodes. The nodes which are all depicted as grey circle indicates such nodes are in sleep mode. The distance between corresponding nodes can be calculated by finding out the nodes position in terms of $\mathrm{X}$ and $\mathrm{Y}$. In this diagram we have assigned node $\mathrm{S}$ as source node and node 1 as destination node.

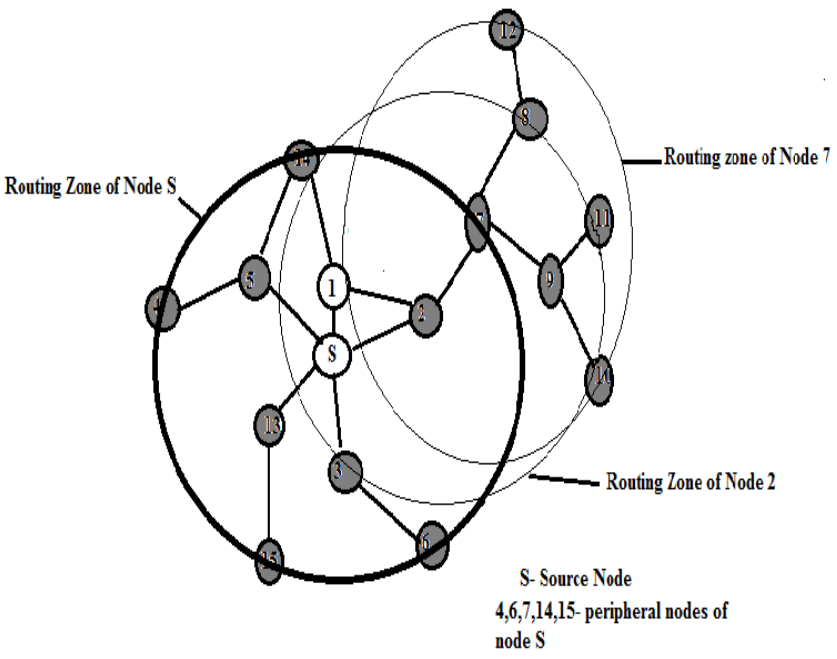

Fig 4: Transmission power control when node 1 acts as Destination

The destination node (Node 1) is nearer to the source node $(\mathrm{S})$ and the distance between node $\mathrm{S}$ and node 1 is almost less than $50 \mathrm{~m}$, so the transmission power of the source node is adjusted based on the predefined power as given in table 1. At initial we have assigned transmission power of $0.2 \mathrm{~J}$ to the nodes. After adjusting the transmission power it consumes $0.09 \mathrm{~J}$ to deliver the data successfully to the destination node (i.e. Node 1). 


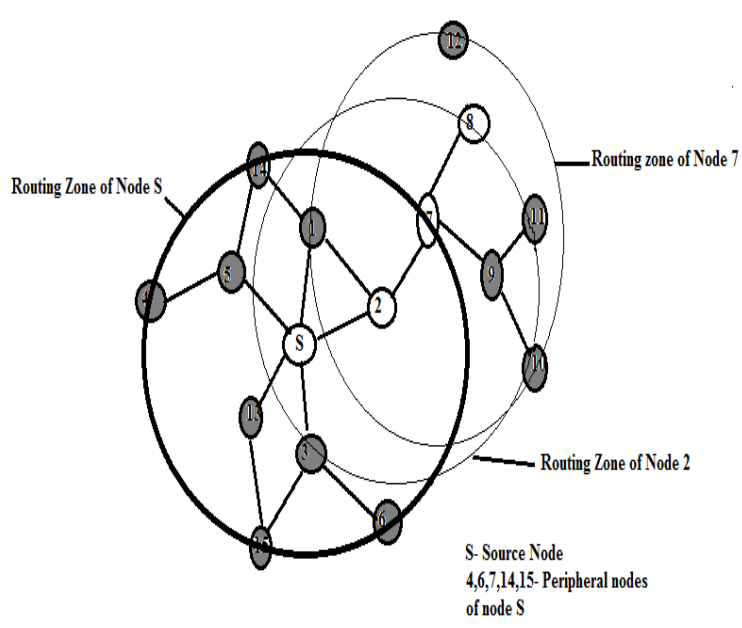

Fig 5: Transmission power control when node 8 acts as destination

Let us take the network given in fig 5 , here node $\mathrm{S}$ is the source node and Node 8 as destination. Once the route(S-78 ) has been established to the node 4 using IERP and IARP, the source node calculate the distance to the node 7 and adjust the transmission power and node 7 calculates the distance to the node 8 and adjusts its transmission power according to the pre-defined power values as given in table I.

\section{ALGORITHM}

Step 1: Periodic Broadcasting of Beacon Messages using NDP (Neighbor Discovery Protocol)

Step 2: Nodes Listen for traffic during Listening time

Step 3: If Traffic exist Switch over to Active Mode for time Ta or else Switch over to Sleep mode for time Ts.

Step 4: During Active Mode Node Check its Destination

$>$ If Destination is covered by its source Zone Region follows Pro Active Mechanism () and use Transmission power control technique ().

$>$ If Destination is not covered by Source Zone Region follow Reactive Mechanism ().

Proactive Mechanism ():

$>$ Routing Table maintenance and Accomplish Packet Delivery process.

Reactive Mechanism ():

$>$ Broadcasting of request to its peripherals using BRP.

$>$ Upon Reception of Request Checking of destination in its routing table and repeat it until it finds destination.

$>$ Once the Destination is found the reply will be send back to source and use transmission power control technique.

Step 5: Transmission power control ():

$>$ Distance calculation and transmission power adjustment using Frris relation.

Step 6: Repeat this until successful delivery of last packet in the queue.

\section{SIMULATION SETUP}

\begin{tabular}{|l|l|}
\hline Parameter & Value \\
\hline Number of Nodes & $20,30,40,50,60$. \\
\hline Grid Area & $800 * 800$ \\
\hline Initial Energy & $100 \mathrm{~J}$ \\
\hline Traffic Model & CBR \\
\hline Transmitter Power & $0.2 \mathrm{~J}$ \\
\hline Receiver Power & $0.1 \mathrm{~J}$ \\
\hline Data Packet Size & 2000 bits \\
\hline Antenna Type & $\begin{array}{l}\text { Omni } \\
\text { Antenna }\end{array}$ \\
\hline MAC Type & IEEE 802.11 \\
\hline Antenna Model & Two Ray Model \\
\hline Simulation Time & 200 sec \\
\hline Listening time $\left(\mathrm{T}_{1}\right)$ & $1 \mathrm{~s}$ \\
\hline Sleep time $\left(\mathrm{T}_{\mathrm{s}}\right)$ & $3 \mathrm{~s}$ \\
\hline Active time $\left(\mathrm{T}_{\mathrm{a}}\right)$ & $20 \mathrm{~s}$ \\
\hline Idle Power & $0.0005 \mathrm{~J}$ \\
\hline Routing Protocol & AZRP \\
\hline Simulation Tool & NS-2 \\
\hline
\end{tabular}

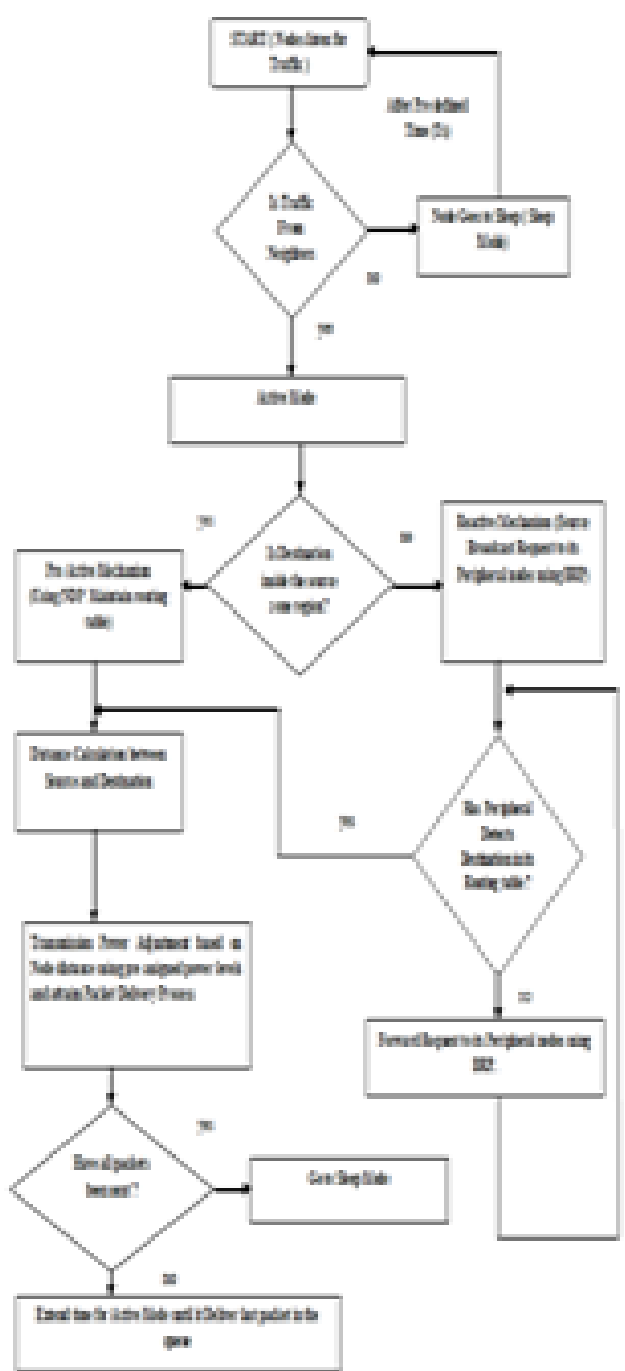

Fig 6: Flow Diagram 
This integrated approach can be simulated in NS-2 environment. The comparison of modified Routing Protocol (AZRP) with Zone Routing Protocol reveals that the modified Zone Routing Protocol has better performance characteristics than ZRP.

\section{RESULT DISCUSSION}

\subsection{Energy}

Energy consumption is the major parameter which decides life time of the network and it contributes more than remaining parameters. The graph shown in fig 7 shows that modified Zone Routing Protocol utilize less amount of energy when compared with the Zone Routing Protocol. AZRP conserves maximum amount of energy by putting the mobile nodes into sleep mode when it is idle and extend its energy conservation during Active mode by taking distance between source and destination into consideration. With the help of this proposed technique we can conserve $48 \%$ of energy under low density and $46 \%$ of energy under high density of nodes when compared with ZRP. As the number of nodes increases, utilization of energy also increases gradually but the conservation of energy in AZRP is more than basic Zone Routing Protocol.

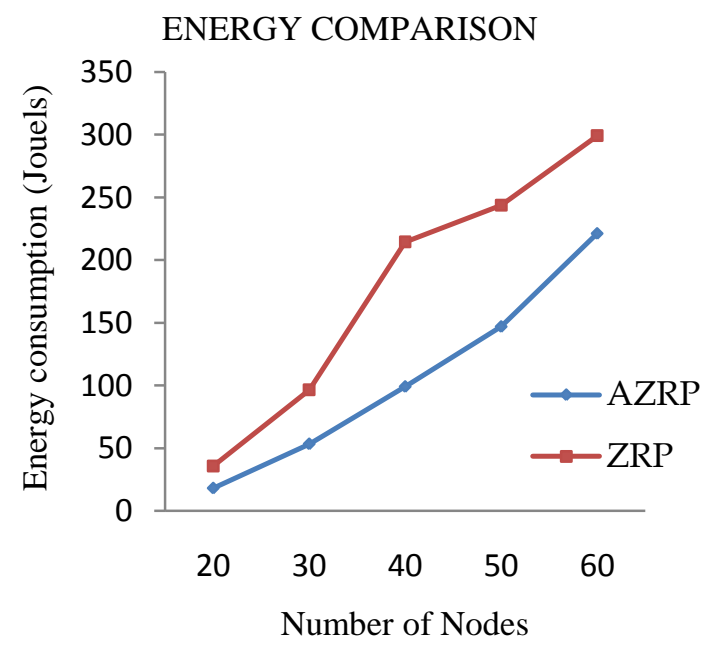

Fig 7: Energy comparison between AZRP and ZRP

\subsection{PDR}

Looking at the Packet Delivery Ratio shown in fig 8, pure ZRP maintains its PDR as an average of $90 \%$ and $83 \%$ in low and high density of nodes respectively. The modified Zone Routing Protocol has the PDR of $87 \%$ and $81 \%$ under low and high density of nodes respectively. It is nothing surprises that reduction of PDR when we use Power saving techniques. The basic reason behind this reduction in PDR is the receiving node could be in sleep mode when the packet arrives and natural packet collision.

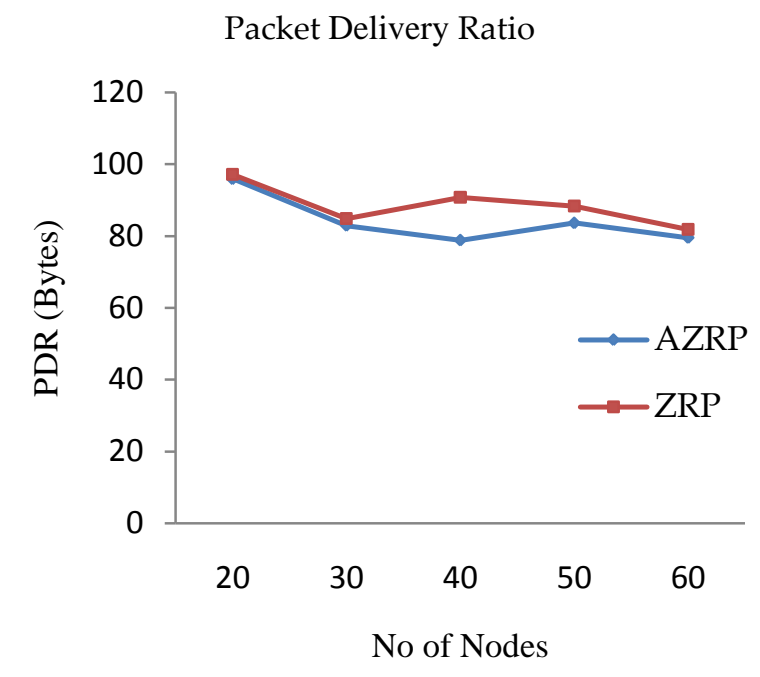

Fig 8: PDR Comparison between AZRP and ZRP

\subsection{Throughput}

An efficient routing protocol should achieve high throughput and low delay. The throughput is always correlated with delay. As the throughput increases the delay will be low. The modified Zone Routing Protocol (AZRP) has higher throughput than basic Zone Routing Protocol and it has less delay as shown in fig 9.The throughput of the network depends upon the traffic density. It is shown that if the node density increases, the throughput of the network slightly decreases but it is comparitively higher than ZRP.

\section{Throughput}

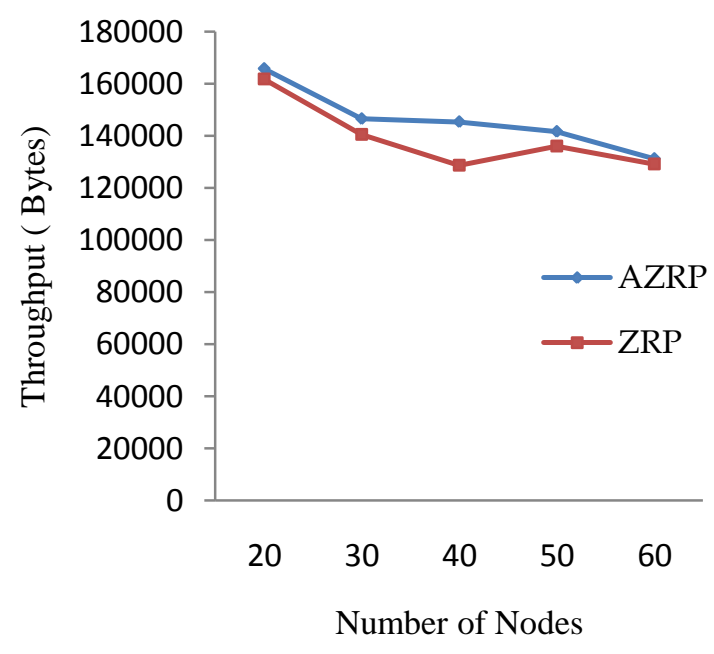

Fig 9: Throughput comparison between AZRP and ZRP

\subsection{Control Overhead}

When AZRP control overhead is compared with ZRP, the overhead of AZRP is slightly higher than ZRP bacause of the re transmission of queries. Once the route has established it will not give much impact on overhead. 


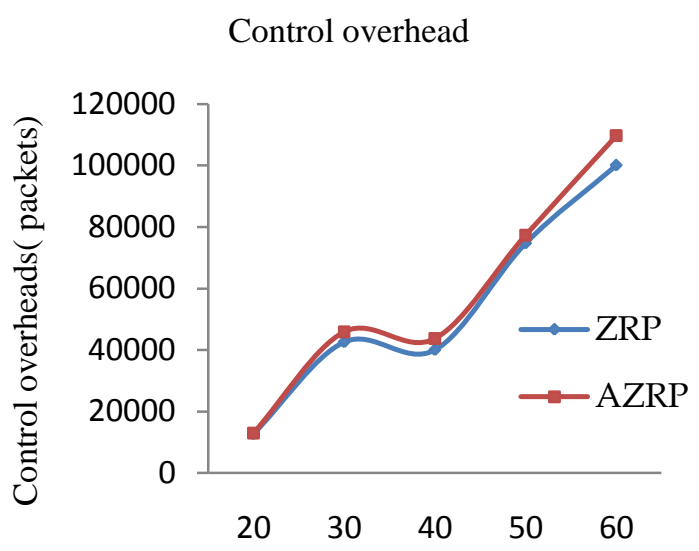

No of nodes

Fig 10: Control overhead comparison between AZRP and ZRP

\section{FUTURE WORK}

This paper proposes a novel way which mainly deals with conservation of battery resource. The basic reason behind the issues of this paper such as reduction in PDR and increase in control overhead is receiving node could be in sleep mode when the traffic flow occurs. Due to this few packets may be dropped and multiple re- transmission causes increase in overhead. This problem can be rectified when it comes to practical by using RF tag to all the nodes in the network. The RF tag helps to wake up the nodes from sleep mode when the traffic flow occurs. This helps to deliver the data packets without any loss and also it reduces multiple re transmission of requests.

\section{CONCLUSIONS}

In this paper we have proposed a novel approach which conserves the energy of the mobile nodes as much as possible by following sleep schedule approach and Distance based energy conservation algorithm. The simulation results were shown which reveals that modified Zone Routing Protocol has better performance which can conserve $48 \%$ of energy under low density of nodes and $46 \%$ of energy under high density of nodes. It ensures that modified ZRP is effectively suitable for wide range of applications.

\section{REFERENCES}

[1] Ravi G. and K. R. Kashwan, "Energy Aware Zone Routing Protocol Using Power Save Technique AFECA" , International Review on Computers and Software, Vol. 8, No. 10, Oct 2013 pp. 2373-2378.

[2] Dinesh RatanGautam , RGPV Bhopal "Enhanced Transmission PowerControl Mechanism based on RSSI for MANET" International Journal of Computer Applications Vol. 28- No.1, Aug 2011, pp. 25-30.

[3] JavadVazifehdan, Venkatesha PrasadR., IgnasNiemegeers“ Energy-Efficient Reliable Routing ConsideringResidual Energy in Wireless Ad Hoc
Networks" IEEE Transactions On Mobile Computing. Vol. 13, No. 2,Feb 2013, pp.434-447.

[4] RavillaDilli,Putta Chandra ShekarReddy,"Energy Management in Zone Routing Protocol (ZRP)" International Journal of Emerging Technology and Advanced Engineering.Vol. 2, No. 5, May 2012.Pp.61-68.

[5] SugunaR., GomathiP., "Energy Conservation in MANET Using Power Saving Protocol BECA/AFECA'IJCSMC, Vol. 2, Issue. 7, July 2013, pp.196 - 199.

[6] MadsDarøKristensen and NielsOlofBouvin, ,"Energy Efficient MANET Routing Using a Combination of Span and BECA/AFECA”Journal of Networks, Vol. 3, No. 3Mar 2008. Pp. 49-56.

[7] SeemaVerma AIM \& ACT, Banasthali University, Rajasthan1, RekhaAgarwal" An Optimized Energy Aware Routing (OEAR) Scheme for Mobile Ad Hoc Networks using Variable Transmission Range"International Journal of Computer Applications. Vol. 45, No.12, May 2012. Pp.18-22.

[8] Jun Zhang, Li Fei, QiangGao, Xiao-Hong Peng"Energy-Efficient Multihop Cooperative MISO Transmission with Optimal Hop Distance in Wireless Ad Hoc Networks" IEEE Transactions on Wireless Communications, Vol. 10, No. 10, Oct 2011.Pp. 3426-3435.

[9] Subhankar Mishra, Sudhansu Mohan Satpathy and Abhipsa Mishra "ENERGY EFFICIENCY IN AD HOC NETWORKS "International Journal of Ad hoc, Sensor \& Ubiquitous Computing (IJASUC) Vol.2, No.1, Mar 2011. Pp. 139-145.

[10] Zygmunt J. Haas, and Marc R. Pearlman, "The Performance of Query Control Schemes for the Zone Routing Protocol" IEEE Transactions on Networking, Vol. 9, No. 4, Aug 2001. Pp. 427-438.

[11] J. Cartigny, D. Simplot, and I. Stojmenovic, "Localized Minimum- Energy Broadcasting in AdHoc Networks," Proceeding of IEEE INFOCOM. Vol. 3, pp. 2210-2217, Mar. 2003.

[12] Laura Galluccio, GiacomoMorabito, and Sergio Palazzo,"Evaluation of a Tradeoff Between Energy Efficiency and Responsiveness of Neighbor Discovery in Self-Organizing Ad Hoc Networks "IEEE Journal On Selected Areas In Communications, Vol. 22, No. 7, Sep 2004. pp. 1167-1182.

[13] Baldev Ram Mali, N.C. Barwar, " The Performance of MANET Routing Protocols considering Impact of Node Density under Different Traffic Patterns "Special Issue of International Journal of Computer Applications on No. 6, Jan2012, pp.26-30. 